\title{
Erratum: "Deformation and failure of a film/substrate system subjected to spherical indentation: Part II. Prediction of failure modes in a thin TiN film deposited on a compliant elastic substrate" [J. Mater. Res. 21, 783 (2006)]
}

\author{
S. Math \\ Department of Mechanical Engineering, Indian Institute of Science, Bangalore-560012, India \\ V. Jayaram ${ }^{\text {a) }}$ \\ Department of Materials Engineering, Indian Institute of Science, Bangalore-560012, India \\ S.K. Biswas \\ Department of Mechanical Engineering, Indian Institute of Science, Bangalore-560012, India
}

(Received 16 January 2008; accepted 22 January 2008)

With reference to the above mentioned article [J. Mater Res. 21, 783 (2006)], we would like to correct two equations and a reference:

1. In the subsection "Criterion for inclined crack initiation" under the section "Analysis"(page 785, column 2, line $15)$, the corrected equation should be read as:

$$
K_{\mathrm{II}}=\left(\frac{2}{\pi l}\right)^{1 / 2} \int_{0}^{l} \tau_{\max }\left(\frac{x}{l-x}\right)^{1 / 2} \mathrm{~d} x
$$

The square root in the term $(2 / \pi l)^{1 / 2}$ is missing in the current article.

2. In the subsection "Criterion for bending crack" (page 786, column 1, line 12), the corrected equation should be read as:

$$
K_{\mathrm{I}}=\left(\frac{2}{\pi l}\right)^{1 / 2} \int_{0}^{l} \sigma_{\mathrm{r}}\left(\frac{x}{l-x}\right)^{1 / 2} \mathrm{~d} x
$$

The square root is missing in the term $(2 / \pi l)^{1 / 2}$, as in the earlier case.

3. The reference numbered 12 should be corrected as

D.J. Green: An Introduction to the Mechanical Properties of Ceramics (Cambridge University Press, Cambridge, UK, 1998), p. 241.

\footnotetext{
a) Address all correspondence to this author.

e-mail: qjayaram@materials.iisc.ernet.in

DOI: $10.1557 / J M R .2006 .0095 \mathrm{e}$
} 